\title{
Crystallization Does It All: An Alternative Strategy for Stereoselective Aza-Henry Reaction
}

Michaela Marčeková ${ }^{\dagger}$, Peter Gerža ${ }^{\dagger}$, Michal Šoral ${ }^{\dagger}$, Ján Moncol ${ }^{\dagger}$, Dušan Berkeš ${ }^{\dagger}$, Andrej Kolarovič๋ and Pavol Jakubec*广

† Faculty of Chemical and Food Technology, Slovak University of Technology, Radlinského 9, 81237 Bratislava, Slovakia

$\$$ Department of Chemistry, Faculty of Education, Trnava University, Priemyselná 4, 91843 Trnava, Slovakia

*e-mail: pavol.jakubec@stuba.sk

Supporting Information 


\section{Table of Contents}

1. Crystallographic data for the reported compounds 3

1.1. Fig. X1 Molecular structure of 5 with the thermal ellipsoids shown at a 50\% probability level 5

1.2. Fig. X2 Molecular structure of $\mathbf{1 1} \cdot 1.5 \mathrm{H}_{2} \mathrm{O}$ with the thermal ellipsoids shown at a $50 \%$ probability level 6

1.3. Fig. X3 Molecular structure of $\mathbf{1 3}$ with the thermal ellipsoids shown at a 50\% probability level 7

1.4. Fig. X4 Molecular structure of $\mathbf{1 6}$ with the thermal ellipsoids shown at a 50\% probability level 8

1.5. Fig. X5 Molecular structure of $\mathbf{2 0}$ with the thermal ellipsoids shown at a 50\% probability level 9

1.6. Fig. X6 Molecular structure of $\mathbf{2 8} \cdot \mathrm{H}_{2} \mathrm{O}$ with the thermal ellipsoids shown at a $50 \%$ probability level 10 


\section{Crystallographic data for the reported compounds}

\section{X-Ray crystallography for the reported compounds}

Data collection and cell refinement of $5,11 \cdot 1.5 \mathrm{H}_{2} \mathrm{O}, 13,16,20$ and $28 \cdot \mathrm{H}_{2} \mathrm{O}$ were made by Stoe StadiVari diffractometer at $100 \mathrm{~K}$ using Pilatus3R 300K HPAD detector and microfocused source Xenocs Genix3D Cu HF (Cu Ka radiation $\lambda=1.54186 \AA$ ). The diffraction intensities were corrected for Lorentz and polarization factors. The structures were solved using SHELXT or SUPERFLIP programs $^{[1,2]}$ and refined by full-matrix least-squares procedure with SHELXL (version 2018/3) for 5, 13, 16 and 20 or CRYSTALS (version 14.61) for $\mathbf{1 1} \cdot 1.5 \mathrm{H}_{2} \mathrm{O}$ and $\mathbf{2 8} \cdot \mathrm{H}_{2} \mathrm{O} \cdot{ }^{[3,4]}$ The multi-scan absorption corrections were applied using Stoe LANA software. ${ }^{[5]}$ Geometrical analyses were performed with SHELXL or CRYSTALS. The structures were drawn with OLEX2.[6] The Flack parameters of both compounds have been calculated using Parsons method. ${ }^{[7]}$

The crystal structure 13 and 20 show disordered groups. The disordered groups of $\mathbf{1 3}$ have been modeled using SHELXL's commands: SAME, EADP and RIGU. The disordered groups of $\mathbf{1 3}$ have been modeled using SHELXL's commands: SAME, EADP, RIGU and SADI. The crystal structure 20 contains disordered solvent molecules which have been removed and masked using OLEX solventmasking procedure.

Crystal Data for (5): monoclinic, space group $P 2_{1}$ (no. 4), $a=5.6491$ (3) $\AA, b=8.0286$ (3) $\AA, c=$ 6.6541(3) $\AA, \beta=104.431(4)^{\circ}, V=292.27(4) \AA^{3}, Z=2, T=100 \mathrm{~K}, \mu($ CuKa $)=5.111 \mathrm{~mm}^{-1}$, Dcalc $=$ $1.597 \mathrm{~g} / \mathrm{cm}^{3}, 13929$ reflections measured $\left(13.742^{\circ} \leq 2 \Theta \leq 141.134^{\circ}\right), 1034$ unique $\left(R_{\text {int }}=0.0160\right.$, $\left.R_{\text {sigma }}=0.0058\right)$ which were used in all calculations. The final $R_{1}$ was $0.0189(I>2 \sigma(I))$ and $w R_{2}$ was 0.0514 (all data). The Flack parameter $\mathrm{x}=0.003(9)$.

Crystal Data for $\left(\mathbf{1 1} \cdot 1.5 \mathrm{H}_{2} \mathrm{O}\right)$ : monoclinic, space group $C 2$ (no. 5), $a=7.6037$ (2) $\AA$, $b=$ 9.6037(2) $\AA, c=15.2325(2) \AA, \beta=98.373(2)^{\circ}, V=1100.48(5) \AA^{3}, Z=2, T=100 \mathrm{~K}, \mu(\mathrm{CuK \alpha})=0.999$ $\mathrm{mm}^{-1}$, Dcalc $=1.383 \mathrm{~g} / \mathrm{cm}^{3}, 15400$ reflections measured $\left(5.864^{\circ} \leq 2 \Theta \leq 142.852^{\circ}\right), 1982$ unique $\left(R_{\text {int }}=\right.$ $\left.0.0366, R_{\text {sigma }}=0.019\right)$ which were used in all calculations. The final $R_{1}$ was $0.0370(I>2.0 \sigma(I))$ and $w R_{2}$ was 0.0971 (all data). The Flack parameter $x=-0.15(3)$.

Crystal Data for (13): orthorhombic, space group $P 2{ }_{1}{ }_{1} 2_{1}$ (no. 19), $a=25.8152$ (3) $\AA, b=$ 16.5527(3) $\AA, c=6.9198(1) \AA, V=2956.91(8) \AA^{3}, Z=4, T=100 \mathrm{~K}, \mu(\mathrm{CuKa})=0.627 \mathrm{~mm}-1$, Dcalc $=$ $1.190 \mathrm{~g} / \mathrm{cm}^{3}, 89861$ reflections measured $\left(6.344^{\circ} \leq 2 \Theta \leq 143.57^{\circ}\right), 5727$ unique $\left(R_{\text {int }}=0.0297, R_{\text {sigma }}=\right.$ $0.0144)$ which were used in all calculations. The final $R_{1}$ was $0.0308(I>2.0 \sigma(I))$ and $w R_{2}$ was 0.0841 (all data). The Flack parameter $\mathrm{x}=-0.02(4)$.

Crystal Data for (16): orthorhombic, space group $P 2{ }_{12121}$ (no. 19), $a=6.6271$ (4) $\AA$, $b=$ 17.34118(11) $\AA, c=26.31426(16) \AA, V=3025.27(3) \AA^{3}, Z=4, T=100 \mathrm{~K}, \mu(\mathrm{CuK \alpha})=0.649 \mathrm{~mm}$ 1 , Dcalc $=1.238 \mathrm{~g} / \mathrm{cm}^{3}, 69897$ reflections measured $\left(6.104^{\circ} \leq 2 \Theta \leq 143.372^{\circ}\right), 5864$ unique $\left(R_{\text {int }}=\right.$ $\left.0.0264, R_{\text {sigma }}=0.0101\right)$ which were used in all calculations. The final $R_{1}$ was $0.0362(I>2.0 \sigma(I))$ and $\mathrm{wR}_{2}$ was 0.0927 (all data). The Flack parameter $\mathrm{x}=-0.38(10)$.

Crystal Data for (20): orthorhombic, space group $P 212121$ (no. 19), $a=7.94784$ (9) $\AA, b=$ 13.00668(13) $\AA, c=29.5402(3) \AA, V=3053.72(6) \AA^{3}, Z=4, T=100 \mathrm{~K}, \mu(\mathrm{CuK \alpha})=0.584 \mathrm{~mm}$ 1 , Dcalc $=1.091 \mathrm{~g} / \mathrm{cm}^{3}, 72981$ reflections measured $\left(5.984^{\circ} \leq 2 \Theta \leq 143.736^{\circ}\right), 5748$ unique $\left(R_{\text {int }}=\right.$ 
$\left.0.0380, R_{\text {sigma }}=0.0184\right)$ which were used in all calculations. The final $R_{1}$ was $0.0501(I>2.0 \sigma(I))$ and $w_{2}$ was 0.1441 (all data). The Flack parameter $x=0.06(9)$.

Crystal Data for $\left(28 \cdot \mathrm{H}_{2} \mathrm{O}\right)$ : triclinic, space group $P 1$ (no. 1), $a=5.8128(2) \AA, b=6.1866$ (2) $\AA, c=$ $6.8551(2) \AA, \alpha=71.868(3)^{\circ}, \beta=72.882(3)^{\circ}, y=62.804(3)^{\circ}, V=204.976(6) \AA^{3}, Z=1, T=100 \mathrm{~K}$, $\mu($ CuKa $)=3.838 \mathrm{~mm}-1$, Dcalc $=1.398 \mathrm{~g} / \mathrm{cm}^{3}, 9471$ reflections measured $\left(13.818^{\circ} \leq 2 \Theta \leq 143.468^{\circ}\right)$, 1092 unique ( $\left.R_{\text {int }}=0.0176, R_{\text {sigma }}=0.0072\right)$ which were used in all calculations. The final $R_{1}$ was $0.0370(I>2 \sigma(I))$ and $w R_{2}$ was 0.0969 (all data). The Flack parameter $x=0.015(1)$.

[1] G. M. Sheldrick, Acta Crystallogr, Sect. A. 2015, A71, 3-8.

[2] L. Palatinus, G. Chapuis, J. Appl. Crystallogr. 2007, 40, 787.

[3] G. M. Sheldrick, Acta Crystallogr, Sect. C. 2015, C71, 3-8.

[4] P. W. Betteridge, J. R. Carruthers, R. I. Cooper, K. Prout, D. J. Watkin, J. Appl. Crystallogr. 2003, $36,1487$.

[5] J. Koziskova, F. Hahn, J. Richter, J. Kozisek, Acta Chim. Slovaca 2016, 9, 136-148.

[6] O. V. Dolomanov, L. J. Bourhis, R. J. Gildea, J. A. K. Howard, H. Puschmann, J. Appl. Crystallogr. 2009, 42, 339-341.

[7] S. Parsons, H. D. Flack, T. Wagner, Acta Crystallogr., Sect. B 2013, B69, 249-259 


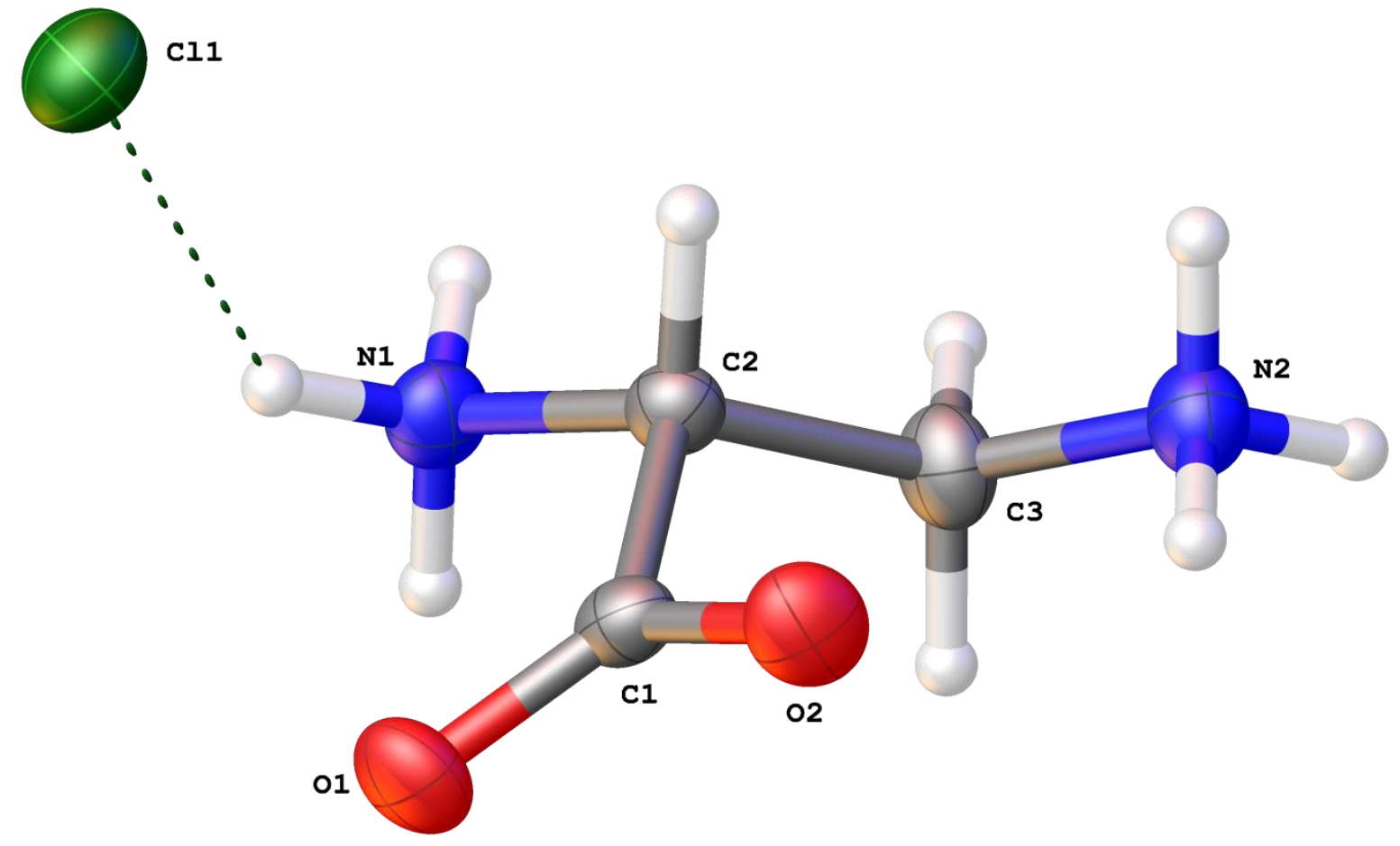

Fig. X1 Molecular structure of $\mathbf{5}$ with the thermal ellipsoids shown at a 50\% probability level. 


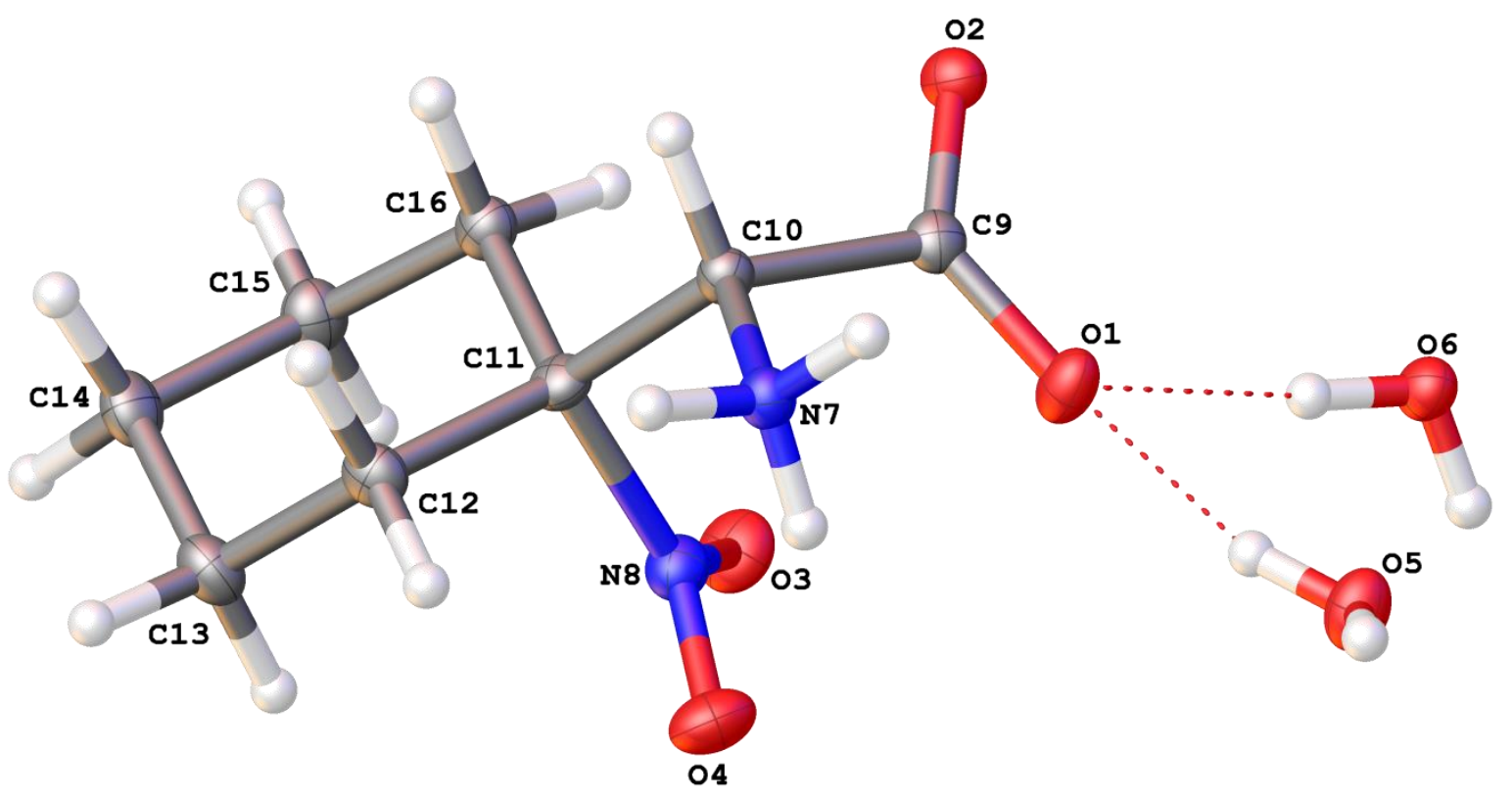

Fig. X2 Molecular structure of $\mathbf{1 1} \cdot 1.5 \mathrm{H}_{2} \mathrm{O}$ with the thermal ellipsoids shown at a $50 \%$ probability level. 


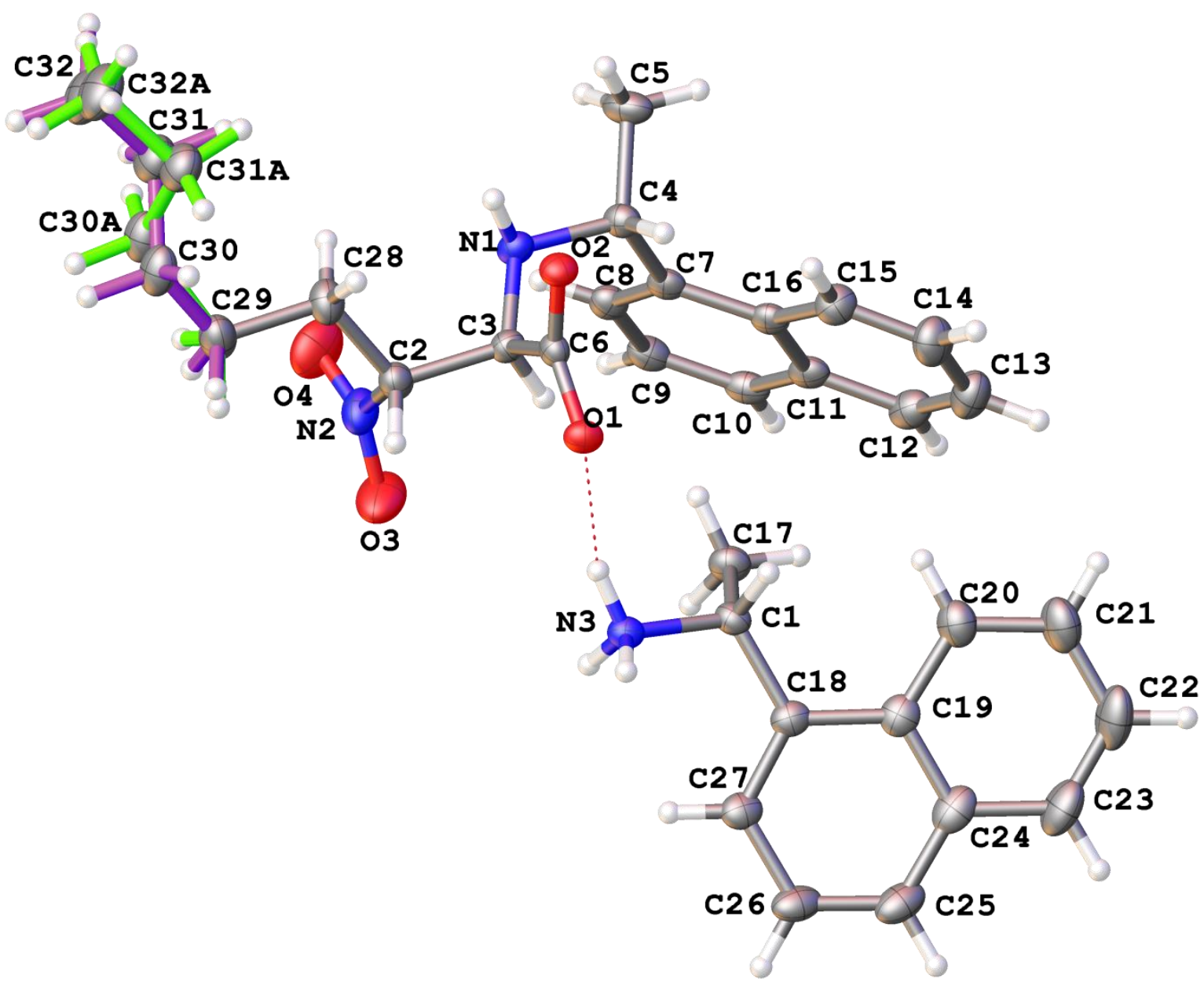

Fig. X3 Molecular structure of $\mathbf{1 3}$ with the thermal ellipsoids shown at a 50\% probability level. The disordered parts of anion are drawn using green and violet lines. 


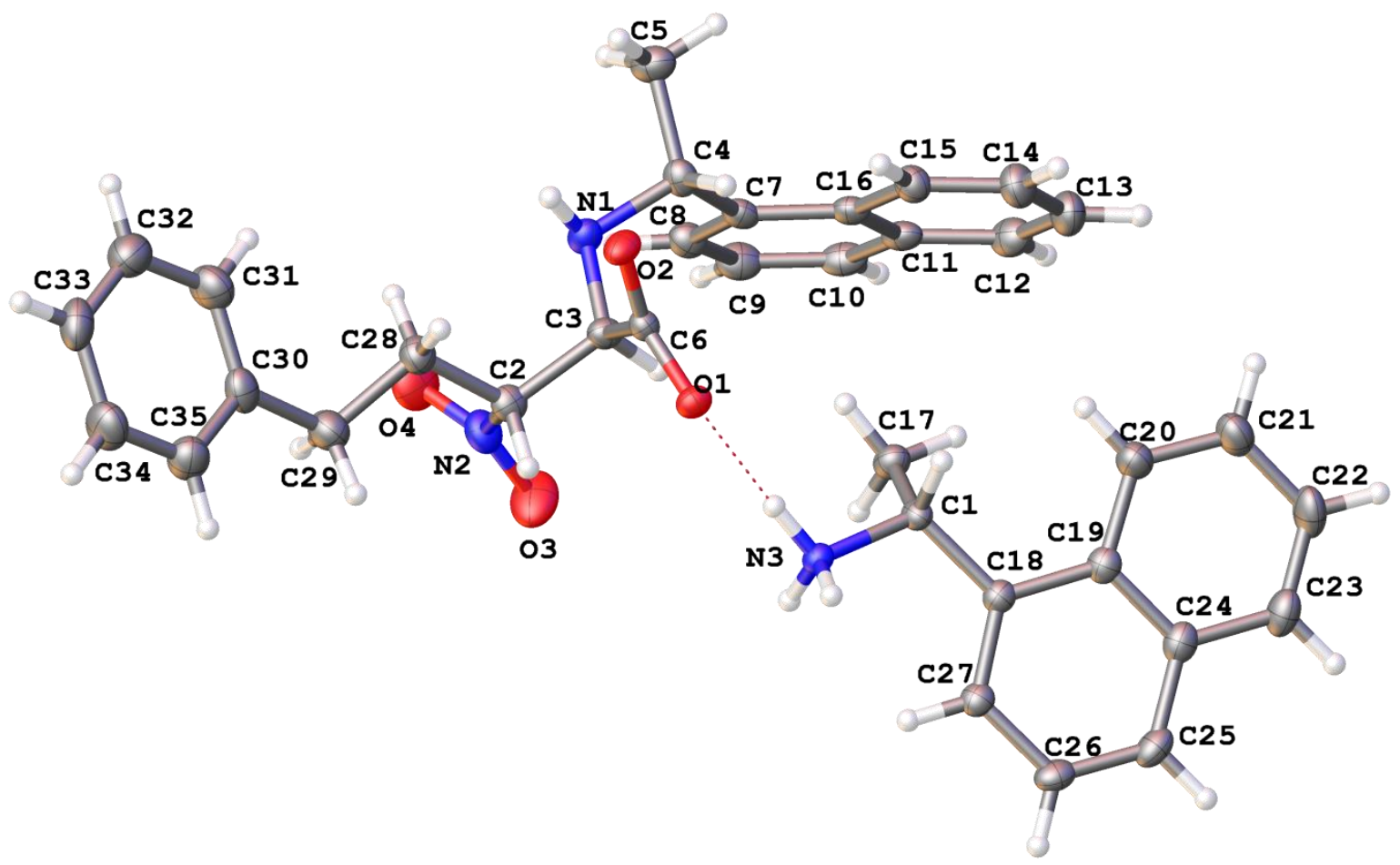

Fig. X4 Molecular structure of $\mathbf{1 6}$ with the thermal ellipsoids shown at a $\mathbf{5 0 \%}$ probability level. 


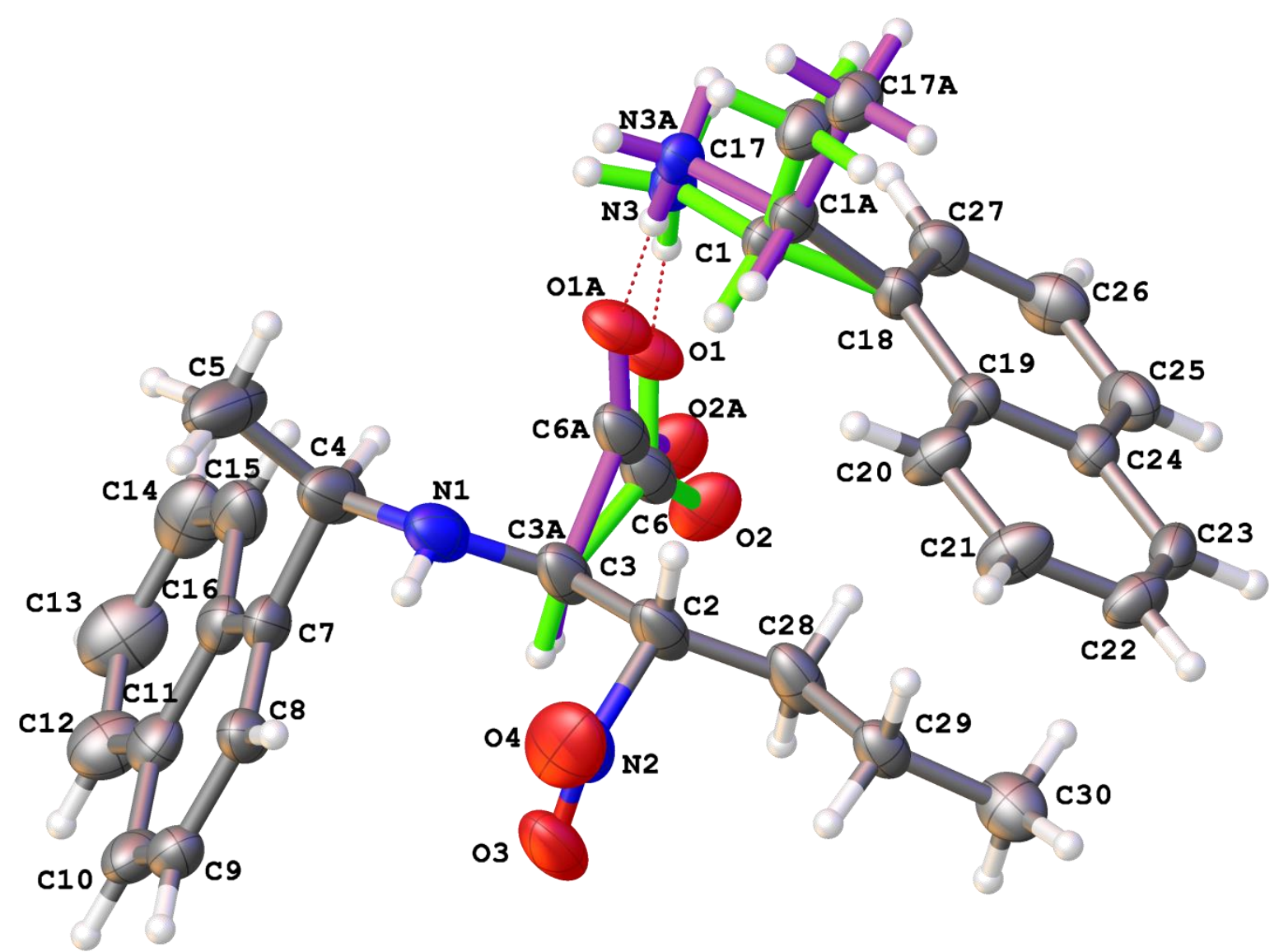

Fig. X5 Molecular structure of $\mathbf{2 0}$ with the thermal ellipsoids shown at a 50\% probability level. The disordered parts of anion are drawn using green and violet lines. 


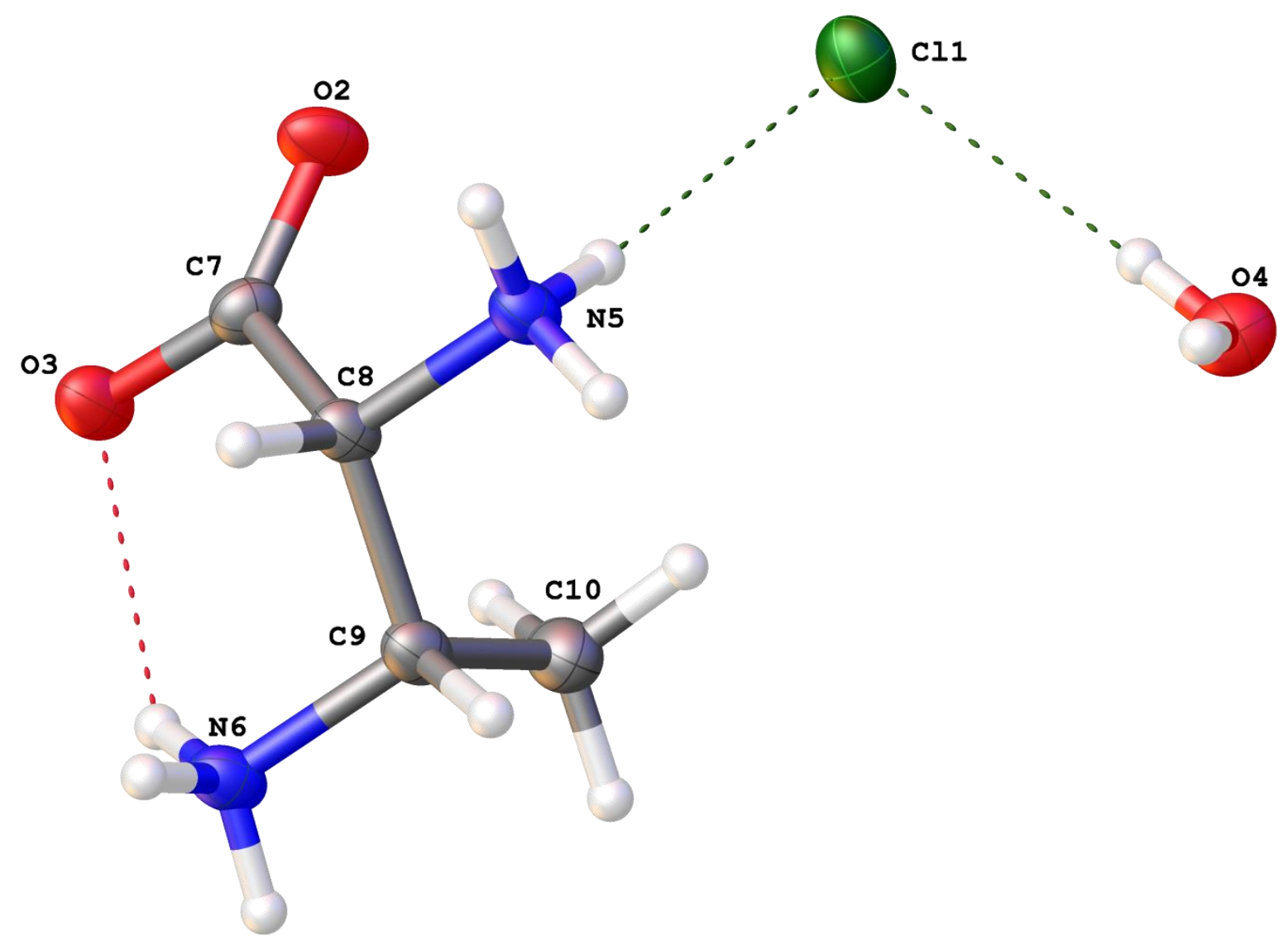

Fig. X6 Molecular structure of $\mathbf{2 8} \cdot \mathrm{H}_{2} \mathrm{O}$ with the thermal ellipsoids shown at a $50 \%$ probability level. 